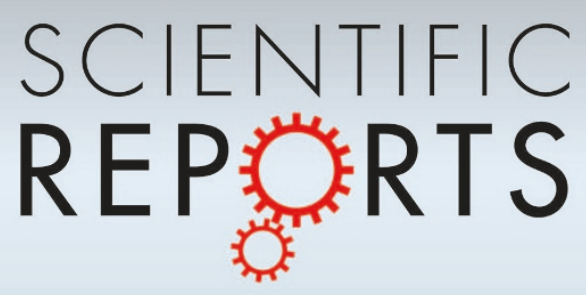

OPEN

SUBJECT AREAS:

COLLOIDS

NANOTECHNOLOGY IN

CANCER

CANCER THERAPY

MAGNETIC PROPERTIES AND MATERIALS

Received

13 May 2013

Accepted

20 September 2013

Published

7 October 2013

Correspondence and requests for materials should be addressed to A.F.B. (bakuzis@ufg. br)

\section{Effect of magnetic dipolar interactions on nanoparticle heating efficiency: Implications for cancer hyperthermia}

\author{
Luis C. Branquinho', Marcus S. Carrião', Anderson S. Costa', Nicholas Zufelato', Marcelo H. Sousa², \\ Ronei Miotto ${ }^{3}$, Robert Ivkov ${ }^{4}$ \& Andris F. Bakuzis'
}

'Instituto de Física, Universidade Federal de Goiás, 74001-970, Goiânia-GO, Brazil, ${ }^{2}$ Faculdade de Ceilândia, Universidade de Brasília, 72220-140, Brasília-DF, Brazil, ${ }^{3}$ Centro de Ciências Naturais e Humanas, Universidade Federal do ABC, Rua Santa Adélia, 166, CEP 09210-170, Santo André, SP, Brazil, ${ }^{4}$ Department of Radiation Oncology and Molecular Radiation Sciences, Johns Hopkins University School of Medicine, Baltimore, MD 21287, USA.

Nanostructured magnetic systems have many applications, including potential use in cancer therapy deriving from their ability to heat in alternating magnetic fields. In this work we explore the influence of particle chain formation on the normalized heating properties, or specific loss power (SLP) of both low(spherical) and high- (parallelepiped) anisotropy ferrite-based magnetic fluids. Analysis of ferromagnetic resonance (FMR) data shows that high particle concentrations correlate with increasing chain length producing decreasing SLP. Monte Carlo simulations corroborate the FMR results. We propose a theoretical model describing dipole interactions valid for the linear response regime to explain the observed trends. This model predicts optimum particle sizes for hyperthermia to about $30 \%$ smaller than those previously predicted, depending on the nanoparticle parameters and chain size. Also, optimum chain lengths depended on nanoparticle surface-to-surface distance. Our results might have important implications to cancer treatment and could motivate new strategies to optimize magnetic hyperthermia.

M agnetic nanoparticle (fluid) hyperthermia has received considerable attention as a potential therapeutic tool for cancer and other diseases. In hyperthermia, the target or diseased tissue is heated to between $43^{\circ} \mathrm{C}$ and $46^{\circ} \mathrm{C}$ for a period of time. With magnetic nanoparticle hyperthermia, the heating is achieved by exposing the region of tissue containing magnetic nanoparticles (heat centers) to an alternating magnetic field, or AMF (usually with frequency between 100 to $500 \mathrm{kHz}$ ). The magnetic nanoparticles dissipate heat from relaxation losses thereby creating localized tissue heating ${ }^{1-8}$. Several loss mechanisms have been proposed to account for the observed heating of magnetic nanoparticles, and depending upon specific experimental conditions, evidence of one or more contribution is often observed ${ }^{7-11}$. As a platform for applications in biology and medicine, magnetic nanoparticle heating (irrespective of mechanism) offers the potential for non-invasive and highly selective therapeutic activity.

To date, much effort has focused on developing synthesis methods to control particle size (and size distribution) or to enhance the particle saturation magnetization. It has recently been demonstrated that other parameters, such as magnetic anisotropy, the mathematical damping factor (due to spin-lattice or spin-spin interaction) in the Landau-Lifshitz-Gilbert equation of motion, particle aggregation and interparticle arrangement are also important ${ }^{4,7,8,12}$.

Generally, theoretical descriptions of ferrofluids are based on models comprising non-interacting particles. This however, is typically not observed experimentally in either free suspension ${ }^{4,13-15}$, gel phantoms ${ }^{12}$, and in biological systems $\mathrm{s}^{16,17}$. In free suspension, interparticle interactions can produce clustering and formation of structures in absence of magnetic fields $s^{4,13-15}$. Such clustering has been shown to affect amplitude-dependent heating behavior that may lead to observed differences among particle formulations ${ }^{7,18}$. In biological systems, nanoparticle-nanoparticle and nanoparticle-protein/cell interactions lead to interstitial and intracellular clustering of particles. Indeed, intracellular localization of nanoparticles generates extreme concentrations of particles as they are packed into endosomes or other subcellular compartments ${ }^{2,16,19}$. While it can be expected that dipoledipole interactions ought to play an important role in heat-delivery application, particularly because nanoparticle concentrations are demonstrably inhomogeneous in biological systems ${ }^{12}$, treatment of this topic is curiously absent from all but a few reported studies ${ }^{4,720-22}$. 
Urtizberea et al. ${ }^{20}$, Presa et al. ${ }^{23}$ and Martinez-Boubeta et al. ${ }^{24}$ found that SLP decreases with increasing concentration of iron-oxide nanoparticles, whereas Martinez-Boubeta et al. ${ }^{22}$ report the opposite trend, a slightly increasing SLP with concentration for low field amplitude (150 Oe, $765 \mathrm{kHz}$ ) for Fe-MgO core-shell particles. When particles were exposed to higher field amplitude (300 Oe) they report decreasing SLP with increasing particle concentrations, and in some cases, a concentration-dependent maximum value of SLP that shifted toward lower concentrations with larger particle size. In addition, Haase and Nowak report simulation results suggesting that the heating power per sample volume has an optimal particle concentration $^{25}$. Tomitaka et al. investigated immobilized nanoparticles that were coated with different molecules ${ }^{21}$. They reported higher SLP values for thicker coatings, implying that SLP decreases with stronger dipole interactions. On the other hand, Dennis et al. reported SLP value of $1075 \mathrm{~W} / \mathrm{g}$ at field amplitude of $1080 \mathrm{Oe}(150 \mathrm{kHz})$ for tightly associated nanoparticles, different from the $150 \mathrm{~W} / \mathrm{g}$ value obtained for the loosely associated nanoparticles. Further Hergt et $\mathrm{al}^{26,27}$ have found evidence of higher hyperthermia efficiency for magnetosomes ${ }^{28}$, which are well known to form chains of nanoparticles that are bound together by a filament of proteins. All these results suggest that nanoparticle organization plays a key role in heating efficiency. Nevertheless, to the authors' knowledge, only Urtizberea et al. and Verde et al. have given detailed consideration to the influence of dipole interactions on heating phenomena by comparing their data with existing interaction relaxation models obtained from the literature ${ }^{7,20}$. Thus, we conclude that the influence of dipole interactions on magnetic heating is still poorly understood.

Here, we propose to model and explain how dipole interactions influence heating properties of self-organized nanostructures, namely nanoparticle linear chain arrangements. Our theoretical model (valid for the linear response regime) will be compared with experimental data obtained from literature and within this work. We show that depending on the dipole interaction strength the specific loss power can either increase or decrease with increasing dipole interactions. The results suggest that experimental conditions, optimal chain size and particle diameter all contribute significantly to heating, thus an optimization of these factors is needed to maximize heating. We hope that this work will motivate exploratory investigation into new strategies to optimize magnetic hyperthermia, which might have a great impact on the biomedical field.

For purposes of discussion here, we assume that (true at least for ferrite-based nanoparticles) the heating process has its origin in hysteresis losses, which are proportional to the area of the hysteresis loop. At low-amplitude fields, where the response is linear (LRT model), the loops are always ellipses and the heating power is given by $^{8}$

$$
P_{m}^{L R T}=\pi \mu_{0} \chi_{0} H_{0}^{2} f \frac{2 \pi f \tau}{1+(2 \pi f \tau)^{2}}
$$

with $\chi_{0}$ the equilibrium susceptibility, $H_{0}$ the field amplitude, $f$ the field frequency and $\tau$ the Néel-Brown relaxation time, written $\mathrm{as}^{29}$ :

$$
\tau=\tau_{0}\left(e^{\sigma}-1\right)\left(2^{-\sigma}+\frac{2 \sigma^{3 / 2}}{\sqrt{\pi}(1+\sigma)}\right)
$$

This expression is valid for uniaxial magnets, at any $\sigma$ value, where $\sigma=K_{e f} V_{p} / k_{B} T$ is a dimensionless anisotropy term and $\tau_{0}=$

$\frac{M_{S}}{2 \gamma_{0} K_{e f}} \frac{\left(1+\alpha^{2}\right)}{\alpha}$ (about $\left.10^{-10}-10^{-8} \mathrm{~s}\right) . K_{e f}$ is the effective uniaxial magnetic anisotropy, $\gamma_{0}$ is the electron gyromagnetic ratio, $M_{S}$ is the saturation magnetization, $V_{p}$ is the particle volume, $k_{B}$ is Boltzmann's constant, $T$ is the sample temperature and $\alpha$ is the damping factor. For uniaxial magnets in the longitudinal case (field applied in the easy axis direction) $\chi_{0}$ is equal to ${ }^{10,30,31}$,

$$
\chi_{0}=\chi_{L}\left[\frac{3 e^{\sigma}}{\sqrt{\pi \sigma} \operatorname{erfi}(\sqrt{\sigma})}-\frac{3}{2 \sigma}\right]
$$

where $\operatorname{erfi}(\sqrt{\sigma})=\left(\frac{2}{\sqrt{\pi}}\right) \int_{0}^{\sqrt{\sigma}} e^{x^{2}} d x$ and $\chi_{L}=\mu_{0} M_{S}^{2} / 3 k_{B} T$. In the superparamagnetic regime $(\sigma \ll 1)$ one recovers the Langevin result, while at the high anisotropy limit it goes to the expected Ising result. Further, because Eq. (1) depends on the relaxation time we can expect a strong influence from dipole interactions ${ }^{32-34}$. At high field values the LRT model is not valid and numerical simulations using the Landau-Lifshitz equation are necessary to understand the response $\mathrm{e}^{7,8}$.

In this study, two magnetic colloid suspensions were characterized. One was a commercial magnetite-based starch-coated nanoparticle suspension (BNF-starch S31009, micromod Partikeltechnologie, GmbH, Rostock, Germany) synthesized by high-pressure homogenization according to the core-shell method ${ }^{35}$. The colloid is dispersed in water at physiological conditions. The other colloid was synthesized by the coprecipitation procedure previously described and consisted of manganese ferrite-based (MNF-citrate) nanoparticles surface-coated with citric acid.

\section{Results}

TEM micrographs of both magnetic fluids revealed particles having different shapes between the samples. A typical TEM picture from the BNF sample is shown in Fig. 1(a), from which it was determined that about $97 \%$ are parallelepiped-shaped, consistent with observations of Dennis et al. ${ }^{4}$ Conversely the MNF particles are more isotropic (Fig. 1(b)). Size distributions estimated from image analysis (using Sturges criteria) are shown in the inset to both figures. In Fig. 1(b) only particle diameters are used to determine size and size distribution (the number of particles counted was $\mathrm{N}=439$ ), whereas for BNF particles in Fig. 1(a) the analysis include minor and maximum lengths as indicated in the picture $(\mathrm{N}=131$, which is lower because of the difficulty in identifying images that clearly reveal both lengths). From the geometrical analysis, we estimated the angle $\beta$ of the parallelepiped-shaped nanoparticles $\beta=81^{\circ} \pm 6^{\circ}$. The solid lines are the best fit to the data using the lognormal size distribution function, $g(D)=\left(\frac{1}{\sqrt{2 \pi} D \sigma_{D}}\right) \exp \left(-\frac{\ln ^{2}\left(\frac{D}{D_{0}}\right)}{2 \delta_{D}^{2}}\right)$, with $D_{0}$ and $\delta_{D}$ the median diameter and size dispersion, respectively. In Fig. 1(a) and $1(\mathrm{~b})$ the values represent median \pm standard deviation.

The room temperature $(\sim 295 \mathrm{~K})$ saturation magnetization of the dried (powder) samples was obtained from magnetization curves (Fig. 1(c)) for both samples at a low-field range. The BNF nanoparticles exhibit a "hard" or higher anisotropy (coercive field of $70 \mathrm{Oe}$ ), while MNF appear "softer" exhibiting less evidence of anisotropy (i.e., no hysteresis), much like a quasi-static superparamagnet. The colloidal particle concentration was determined from magnetometry analysis.

The hyperthermia field analysis of the most concentrated sample indicates that SLP scales quite well with the square of the field up to amplitude values of 200 Oe (see supplementary material - Figure S1), in accordance with the LRT model. As a consequence, we investigated the concentration dependence within the low field limit, i.e. 133 Oe and at frequency $500 \mathrm{kHz}$. Details of the magnetic hyperthermia experiments and setup have been previously described in Ref. 7.

The SLP estimated from heating data decreases with increasing particle concentration for both samples (Fig. 1(d)), suggesting that stronger dipolar interactions decrease magnetic heating efficiency. The results also show that, in the low-field regime the "softer" or low-anisotropy particles heat more efficiently than harder or highanisotropy particles ${ }^{7,36}$. Note that one cannot mix the heating efficiency concept with temperature variation. In all cases we found 

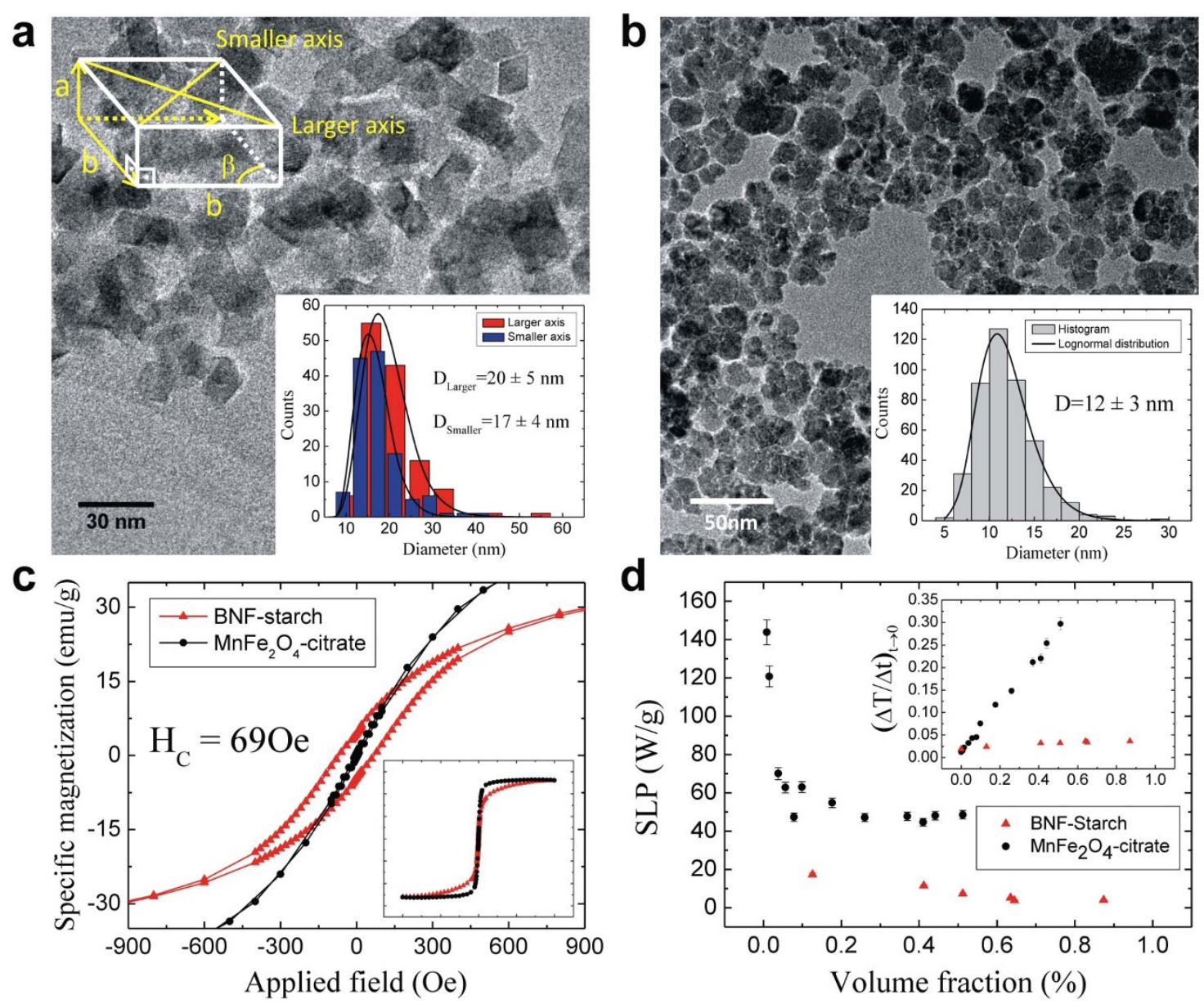

Figure 1 Nanoparticle characterization and hyperthermia particle concentration dependence. (a) TEM picture of the BNF sample. The inset shows the minor and major axis length distribution obtained from the TEM pictures. (b) TEM picture of the MNF sample. The inset shows the size distribution obtained from the TEM pictures. (c) Magnetization curves of both samples. The inset shows the same at a large field range. (d) SLP as function of particle volume fraction for both samples at 133 Oe. The inset shows the heat rate as function of particle concentration for both samples.

temperature variation increasing with increasing particle concentration (see inset of Fig. 1(d)).

It is well established with a variety of methods that in magnetic colloids mean chain length increases with increasing particle concentration ${ }^{13-15,37,38}$. Among the methods used to characterize chain formation, electron magnetic resonance (EMR) has been used to correlate SLP data more directly with chain length ${ }^{14}$. This technique enables study of averaged properties of samples containing large particle concentrations and with rapid data acquisition, thus providing distinct advantages over other methods such as magnetooptical $^{15,37,38}$ and cryogenic-TEM ${ }^{39}$. This is possible because the electron magnetic resonance is sensitive to changes in the effective field, which in this system are due (mainly at low concentrations) to the dipolar field contribution from neighbor magnetic particles forming the chain.

The resonance condition, within the Smit-Beljers approach ${ }^{40}$, is given by

$$
\omega=\frac{\gamma \sqrt{1+\alpha^{2}}}{M_{s} \sin \theta_{0}} \sqrt{\left(F_{\theta \theta}\right)_{0}\left(F_{\phi \phi}\right)_{0}-\left(F_{\theta \phi}\right)_{0}^{2}}=\gamma 1+\alpha^{2} H_{e f},
$$

which depends on the free energy density $(F)$ of the system. $\gamma$ is the gyromagnetic ratio, $\omega$ is the angular microwave frequency, $F_{i j}$ corresponds to the second derivative of the free energy density evaluated at the magnetization equilibrium position, $H_{e f}$ the effective magnetic field and $\theta$ and $\phi$ the angles indicated in the inset of Fig. 2(a).

In general taking into account the dipolar interactions can be very cumbersome. However, for particular configurations such calculations can be performed practically. For symmetrical coherent reversal, the magnetic moments of neighbor nanoparticles are assumed to be at the same angle with respect to the chain anisotropy axis ${ }^{41}$. Considering that the center to center distance between nanoparticles is $r=D_{p}+$ $d_{s-s}$, where $D_{p}$ is the mean diameter and $d_{s-s}$ the surface-to-surface mean distance between nanoparticles forming the chain, we obtain the following free energy (in CGS units) for a nanoparticle in a coherentlike chain configuration ${ }^{14}$

$$
F_{\text {tot }}=-\left[\frac{3 V_{P} M_{S}^{2}}{\left(D_{\text {Larger }}+d_{S-S}\right)^{3}} \sum_{i=1}^{Q} \frac{(Q-i)}{Q(i)^{3}}+2 \pi M_{S}^{2}\left(N_{\perp}-N_{\|}\right)+K\right]
$$$$
\sin ^{2} \theta \sin ^{2} \varphi-M_{S} H \sin \theta \sin \varphi,
$$

where $\mathrm{Q}$ is the chain length (see the supplementary material for SI units). The first term corresponds to the dipole-dipole interaction term of a chain formed of Q particles, the second term is demagnetization contribution, the third the effective magnetic anisotropy (assumed uniaxial), while the last represents the Zeemann interaction. Note that the contribution due to entropic effects in the free energy is typically neglected in EMR analysis. Minimization of the free energy gives us $\theta_{0}$ and $\phi_{0}$ equal to $\frac{\pi}{2}$. Here we also considered that the anisotropy axis of the nanoparticles in the chain is in the field direction due to the possibility of nanoparticle orientation inside the fluid, and we neglect chain-chain interactions because of the highly diluted samples. (A detailed calculation for coherent and fanning dimers, i.e. $\mathrm{Q}=2$, can be found in the supplementary material - section S2). Using $F_{t o t}$ in 

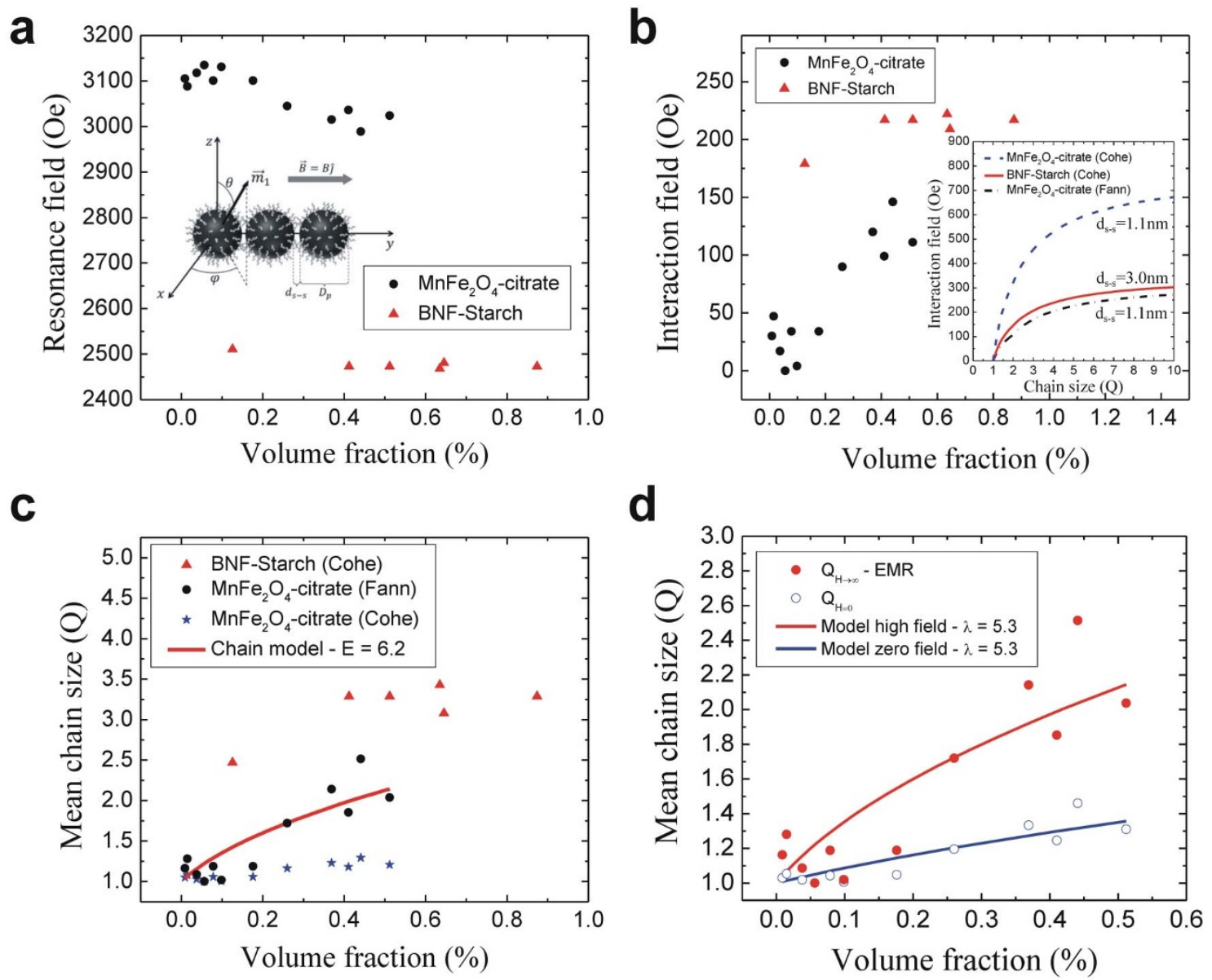

Figure $2 \mid$ Magnetic resonance chain determination. (a) Electron magnetic resonance fields as function of particle volume fraction for both colloidal samples. The inset shows a schematic representation of the chain of nanoparticles in the laboratory system (b) Interaction field as function of particle volume fraction for both samples. The inset shows the theoretical interaction field as function of chain size for both samples in the fanning and coherent configurations. (c) Mean chain size as function of particle volume fraction for both samples, MNF (coherent and fanning) and BNF (coherent). The solid line is the best fit of the MNF fanning data using the chain model discussed in the text. (d) Mean chain size as function of volume fraction. Solid circles correspond to the EMR (high field) estimation, while open symbols are the corrected values at zero field condition. The solid lines are related to the same fit of item (c).

the Smit-Beljers relation one can find the resonance field condition from which is shown that the interaction field is

$$
H_{R}^{\text {mon }}-H_{R}^{\text {chain }}(\phi)=H_{\text {int }}=\frac{6 V_{P} M_{S}}{\left(D_{\text {Larger }}+d_{S-S}\right)^{3}} \sum_{i=1}^{Q} \frac{(Q-i)}{Q(i)^{3}}
$$

Therefore if the resonance field of isolated nanoparticles $\left(H_{R}^{\text {mon }}\right)$ can be calculated and the resonance field of the agglomerate is known (EMR field), one can obtain the interaction field, which on the other hand can be used to extract information about the chain size $(Q)$. A similar calculation for the fanning configuration can be found in Ref. 14.

Fig. 2(a) shows the EMR field as function of particle volume fraction for both samples (EMR spectra can be found in the supplementary material - Figure S3). As expected, due to chain formation, one observes a decrease in the EMR field for increasing concentration. Further, $K_{e f}$ of the soft-ferrite was estimated directly from the lowest resonance field data (see Fig. 2(a)) revealing $2.5 \times 10^{4} \mathrm{erg} / \mathrm{cm}^{3}$, while for the hard-like we used the coercive field approach of Ref. 36, from which we found $8.2 \times 10^{4} \mathrm{erg} / \mathrm{cm}^{3}$. (A similar value was also obtained using the sum of distinct anisotropy contributions, i.e. using an estimated value of magnetite for spherical samples plus a shape anisotropy term, assuming, for simplicity, an ellipsoidal-shape with eccentricity given by the ratio of the larger to the lower length values of the inset of Fig. 1(a)). From this analysis we were able to obtain $H_{R}^{\text {mon }}$ and, as a consequence, calculate the interaction field for both samples (see Fig. 2(b)). It is important to notice that the $H_{\text {int }}$ is higher than the anisotropy field, for both samples, therefore weaklyinteracting relaxation models ${ }^{31,33}$, that predict lower anisotropy or lower susceptibility values for increasing interaction strength, might not be applicable to our data.

The inset of Fig. 2(b) shows the theoretical interaction field as a function of chain size for both samples considering coherent and fanning configurations. For BNF particles only coherent rotation is possible. Here we used a surface-to-surface distance of $1.1 \mathrm{~nm}$ for MNF (obtained from magneto-optical analysis - see supplementary material - Figure S4) and $3 \mathrm{~nm}$ for BNF, which corresponds to an average value of typical molecules used for coating. Note that this value corresponds to a compressible length due to chain formation, while other experimental results suggest the extended molecule length for the starch layer is $20 \mathrm{~nm}^{4}$.

Fig. 2(c) shows the mean chain size concentration dependence for both samples. Note that this was done comparing the theoretical calculations of the inset of Fig. 2(b) with the data of Fig. 2(b). As expected, increasing the particle concentration increases the mean chain size. We also performed polydisperse Monte Carlo simulations for the MNF-sample, which confirmed the existence of small agglomerates even at the low particle concentration range (see supplementary 
material - Figure S5). Our MC simulations corroborate our FMR data analysis, enabling us to relate the SLP decrease with particle concentration to the increase in chain size (see Fig. 3 (a)).

One should keep in mind, however that the experimental conditions for EMR and hyperthermia are different (see supplementary material - section S3). This means that the chain value extracted from EMR should be regarded as approximate. Further, according to the literature $\mathrm{e}^{14,38}$ the dependence of mean chain size on the volume fraction is given by, $\langle Q\rangle=2 \varphi e^{E} /\left(\sqrt{1+4 \varphi e^{E}}-1\right)$ with $\varphi$ the particle volume fraction and $E$ a dimensionless energy parameter between particles in the chain. The solid line in Fig. 2(c) is the best fit of the MNF sample using this equation, which gave $E=6.2$. According to the flexible chain model, this parameter for zero field condition, reads $E_{H=0}=2 \lambda-\ln \left(3 \lambda^{3}\right)$, whereas at the high field limit $E_{H \rightarrow \infty}=2 \lambda-\ln \left(3 \lambda^{2}\right)$ (see supplementary material - section S6 for more details). Note that the EMR field occurs close to $0.3 \mathrm{~T}$, while the ac hyperthermia field amplitude is on the order of $0.013 \mathrm{~T}$. So, one might consider the EMR chain estimation as the high field limit case. Fig. 2(d) shows the volume fraction dependence of the EMR (high field) chain, and the estimated value for the zero field condition. The line for the EMR case corresponds to the best fit of the data $(\lambda=5.3)$, which should be interpreted as an average value. Considering that the equation above is valid for each point one can recalculate from the
EMR (high field) chain size the result for the zero field chain (see open symbols). From now on, we will consider this value as the mean chain size of the magnetic colloid at the low field amplitude hyperthermia condition.

Fig. 3(a) shows the SLP as function of chain size for both samples where we considered the fanning configuration for the Mn-sample (and the zero field chain size estimation). In both cases (see symbols) one observes a decrease of SLP with increasing chain length, Q. Note that the lower SLP value of the BNF sample was expected since $\sigma_{0}$ has a quite high value (greater than 6), when compared to MNF. In this case, at the low field range, increasing anisotropy accompanies a decreased SLP. While, above a critical field (high field regime), the opposite behavior is found ${ }^{7}$. On the other hand the Mn-ferrite sample has a low anisotropy that was expected to increase with increasing particle concentration because the efficiency analysis $\left(\mathrm{SLP} / \mathrm{H}^{2}\right)$ revealed that the MNF-nanoparticles were at the low-barrier regime (see supplementary material - section $\mathrm{S} 1$ ). Note that the two highest SLP values correspond to the lowest particle concentrations, which is very close to our experimental precision. Though this is true, larger mean chain length in comparison with higher concentrations is related to coating layer desorption, as observed experimentally ${ }^{13}$.

Polydisperse calculations using the sample parameters and a fixed $\tau_{0}$ value, can be performed to analyze the observed SLP values. For
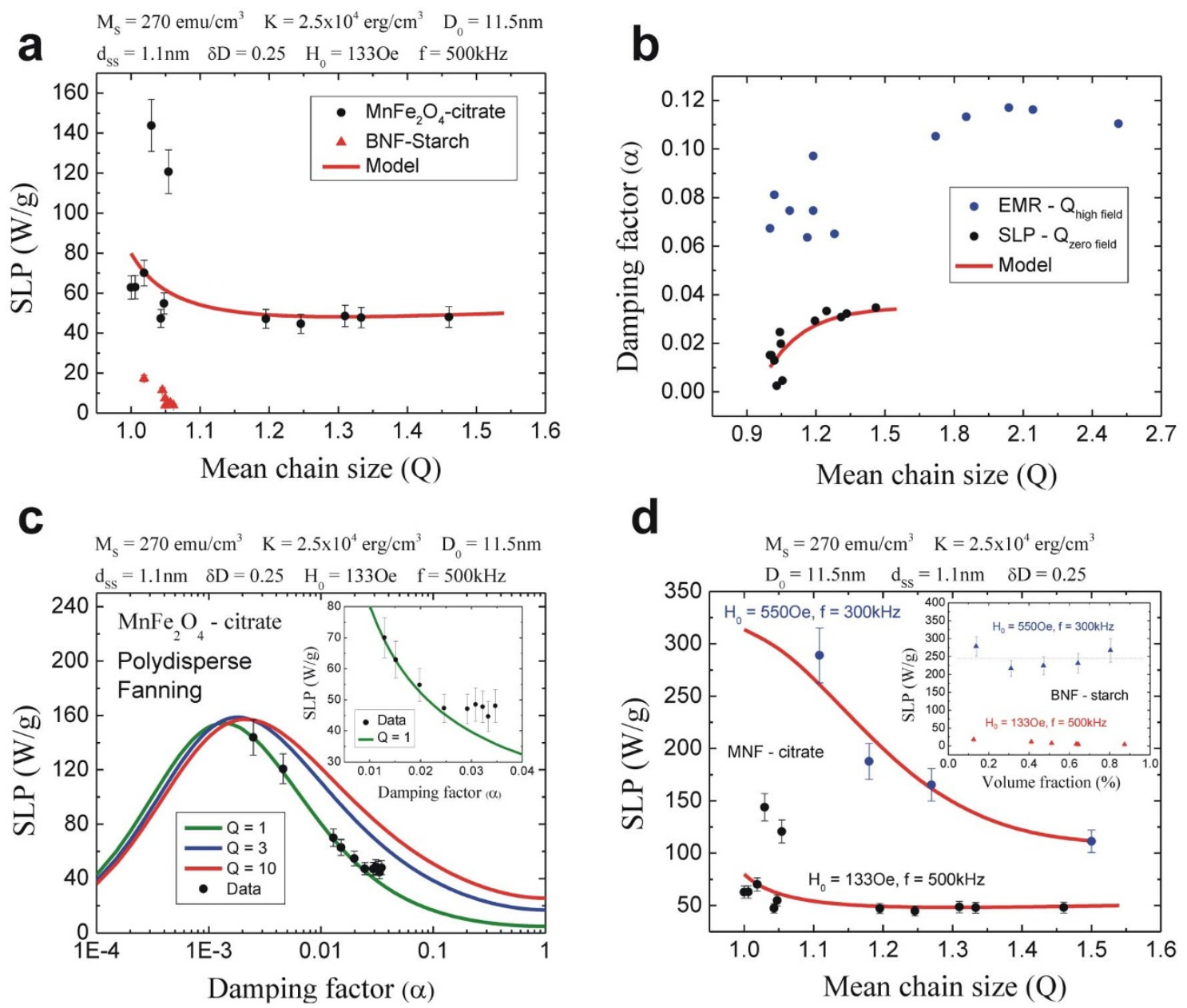

Figure 3 Hyperthermia nanoparticle chain dependence. (a) SLP as function of chain size for both samples. Symbols represent experimental data while the solid line correspond to the theoretical model considering different damping factor values, as discussed in the text. (b) Damping factor as function of chain size. Blue circles correspond to EMR estimation, while black circles to the SLP procedure. Solid line corresponds to the best fit for SLP estimation.

(c) SLP as function of damping factor for the cases $Q=1,3,10$. Symbols represent the experimental data. The inset shows the data, at a linear scale, while the line is the case with $\mathrm{Q}=1$. (d) SLP as function of chain size for the MNF sample at different experimental conditions. Black circles correspond to data at low field and frequency of $500 \mathrm{kHz}$, while black circles are high field amplitude data for a lower frequency $(300 \mathrm{kHz})$. The inset shows the data for the BNF-starch sample. 
BNF particles, in our calculations, we used a volume size distribution. One possible interpretation of the BNF data corresponds to $\tau_{0}$ in the range of 3.6-5.0 $\times 10^{-5} \mathrm{~s}$, which would imply the $\alpha$ value of $6 \times 10^{-6}$. For MNF $\tau_{0}$ in the range of 7.7-9.0 $\times 10^{-7} \mathrm{~s}$ could also give SLP values of the order obtained, which results in a $\alpha$ value of $1 \times 10^{-4}$. Indeed, not only the $\alpha$ values are not in the range typically observed experimentally on such systems but, also, according to recent $a b$ initio calculations, they predict non-physical damping values ${ }^{44}$. In the next section we offer a solution to this problem. Before discussing these, however it is useful to discuss further the dipolar theoretical model.

\section{Discussion}

The theoretical model is based on the fact that dipolar interactions in linear nanoparticle chain arrangements contribute an additional term to the (effective) magnetic anisotropy, as observed in Eq. (5). Note that this has important consequences. Take for instance, a typical $\tau_{0}$ value of $10^{-9} \mathrm{~s}$. Using the MNF nanoparticle parameters this will correspond to a damping value of 0.3. According to eq. (1) maximum heating will occur for $\sigma_{0}$ close to 7. A similar conclusion has been reported from numerical anaylsis (see Ref. 45). (For details of $\tau_{0}$ and the damping factor influence on optimum anisotropy see the supplementary material - Figure S7). The fact that dipolar interactions influence the effective magnetic anisotropy, $\sigma_{e f}=\sigma_{0}+\sigma_{\text {dip }}$, means that one should expect a shift of the dimensionsless optimum anisotropy $\sigma_{0}$ towards lower values, if this interaction increases the effective anisotropy. Therefore, lower optimum particle sizes should be expected in this case. In this section we discuss the influence of the dipolar interaction on the heating efficiency.

Indeed as size dispersion $\left(\delta_{D}\right)$ strongly affects heating efficiency, we also integrate $P_{m}^{L R T}$ considering a log-normal size distribution. Fig. 4(a) shows a typical theoretical calculation considering the experimental saturation magnetization and magnetic anisotropy $(K)$ values (of the spherical soft-ferrite) for a typical nanoparticle coating layer length $(1 \mathrm{~nm})$. The damping factor value was 0.1 , which is used to calculate $\tau_{0}$, with a size dispersion of $\delta_{D}=0.25$ (for polydisperse cases). The solid line represents results obtained from calculations for the monodisperse case using the longitudinal susceptibility (Eq. (3)). The peak drifts to higher $\sigma$ values increasing the damping value (see supplementary material - Figure S7). Q = 1 corresponds to the non-interacting (monomer, or single particle) case. As expected size dispersion lowers the maximum SLP values ${ }^{11}$. In addition, it is found that longer chain length correlates with lower SLP but the optimum dimensionless anisotropy parameter $\sigma_{0}$ is obtained at lower values. This clearly indicates that in general dipole interactions decrease the heating efficiency. Another relevant point to mention is that at the low barrier regime (small $\sigma_{0}$, i.e. low size) the interaction effect may be difficult to identify in polydisperse systems when compared to the non-interacting case.

In Fig. 4(b) we show SLP as function of chain size (Q) considering different particle sizes and damping values. This monodisperse calculation clearly indicates an extremely rich behavior depending on the particle characteristics. One can observe SLP decreasing or increasing for longer chains. Optimum chain sizes can be found, and these results provide a plausible explanation for otherwise apparently contradictory results recently reported ${ }^{20-23}$.

The effect of the nanoparticle coating layer thickness is shown in Fig. 4(c) for monodisperse and in Fig. 4(d) for polydisperse cases. Chain formation increases SLP in a monodisperse system of magnetic nanoparticles reaching a maximum value for $Q \sim 2$ to 6 , depending upon coating thickness. Also, it is worth noting that SLP remains high for longer chains (i.e, $Q>6$ ) with thicker coatings. In stark contrast, however is the polydisperse case (Fig. 4(d)) for which chain formation degrades SLP, regardless of particle coating thickness. Our findings are in agreement with the recently published results of Tomitaka et al. ${ }^{21}$.
The dynamic hysteresis curve can be obtained from this model by noting that the time dependence of the magnetization in LRT is given $\mathrm{by}^{8}$.

$$
M(t)=\frac{\chi_{0}}{\sqrt{1+(2 \pi f \tau)^{2}}} H_{0} \cos (2 \pi f t+\varphi),
$$

where $\varphi$ corresponds to the phase difference between the applied magnetic field $\left(H(t)=H_{0} \cos (2 \pi f t)\right)$ and the magnetization. This term is responsible for the appearance of dynamic hysteresis i.e. heating of quasi-static superparamagnets, and can be found from $\tan (\varphi)=2 \pi f \tau$. Fig. 4(e) shows results of calculations for different chain sizes and surface particle distances when particle size is monodisperse. It is worth noting again that effects of chain length and coating thickness are most pronounced for a monodisperse system of particles. Again, our simulations are similar to the ac hysteresis experimental data of Tomitaka et al. ${ }^{21}$.

Often the goal of magnetic nanoparticle synthesis for hyperthermia applications is to develop methods that synthesize monodisperse nanoparticles. In the low field regime, it was found that optimum dimensionless anisotropy parameters shift toward lower values. In fact, assuming a constant intrinsic magnetic anisotropy, one can easily investigate how the optimum nanoparticle size is influenced by chain formation. In Fig. 4(f) we show the ratio between optimum diameters $(D(Q) / D(Q$ $=1)$ ) as function of chain size for distinct surface to surface particle distances for a typical polydisperse system $\left(\delta_{D}=0.25\right)$. Note that the predicted optimum size can change by around $30 \%$ depending on the particle parameters. This calculation corresponds to the coherent chain case. For the fanning chains this value is around $20 \%$ (see supplementary material - Figure S2). Note that the simulations may have overestimated the SLP values for large particle sizes and high field amplitude values. Nevertheless, the calculations do not influence the optimum diameter chain size dependence.

At this point, we are tempted to stop the SLP chain size dependence analysis, because the observed experimental results can be explained qualitatively by the model. To perform a quantitative SLP analysis, however one needs to further improve the discussion. First, we notice that the $\tau_{0}$ values estimated are inconsistent with both experimental and theoretical analysis ${ }^{7,45}$. As a consequence we estimated $\alpha$ from EMR, by combining the EMR field condition with the EMR linewidth for an isotropic ellipsoid, $\delta H=2 \alpha H_{e f}{ }^{42}$. In Fig. 3(b) blue circles correspond to this estimation where it was observed that $\alpha$ increases with increasing concentration. This behavior has been observed by others ${ }^{33}$. The value obtained however does not agree with the experimental hyperthermia data because it predicts lower SLP values. The higher estimated $\alpha$ value was expected due to more complex contributions to $\delta H$, namely inhomogeneous and extrinsic two-magnon scattering terms ${ }^{43}$, as well as frequency differences between EMR and SLP. As a consequence, we estimate $\alpha$ directly from SLP, for each data point, knowing that MNF is at the low barrier regime. Spheres corresponding to this analysis were fitted (see line) to better analyze the SLP chain dependence. Note the same trend in the $\alpha$ chain dependence from SLP when compared to EMR. Also, the values are close to first principle calculations and show that the interaction field is equivalent to a decrease in temperature ${ }^{44}$. The solid line in Fig. 3(a) corresponds to this model, giving a $\tau_{0}$ ranging from $0.3-6.5 \times 10^{-9} \mathrm{~s}$. Indeed, in our view, the quantitative agreement is quite satisfactory because more complex nanoparticle arrangements might occur in the magnetic colloids.

Further, in Fig. 3(c) SLP as function of damping parameter for different fanning chain sizes of the MNF sample are shown. Solid symbols correspond to our data analysis, while the inset shows the same data at a linear scale. In this case the solid line corresponds to the monomer (isolated nanoparticle) case. For larger damping values (higher concentrations) deviations are related to chain formation, i.e. to the dipolar effect. Further, there are two possible ranges of 

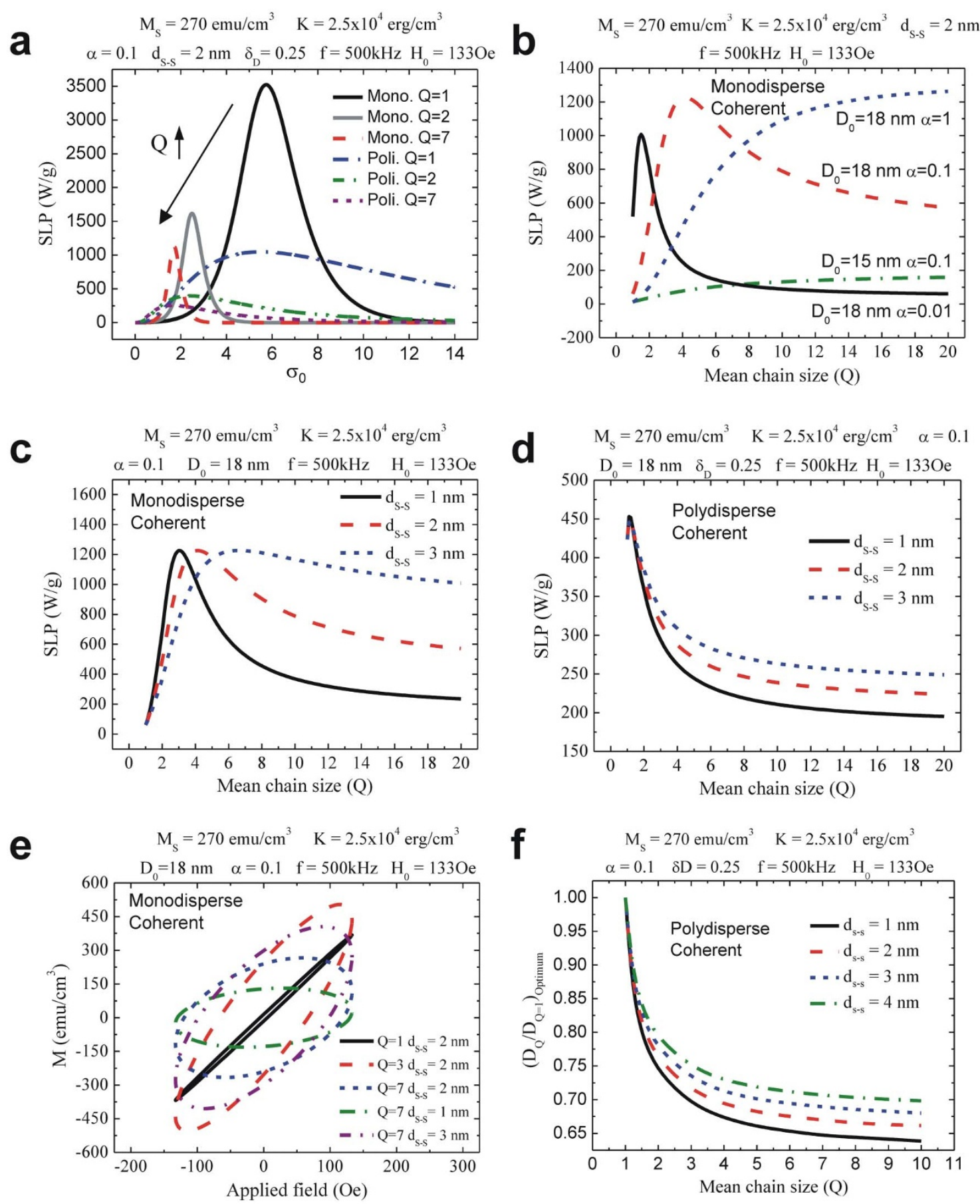

Figure $4 \mid$ Chain theoretical model calculations. (a) SLP as function of dimensionless anisotropy term for mono-disperse and poly-disperse samples considering the non-interacting case $(\mathrm{Q}=1)$ and different chain sizes $(\mathrm{Q}=2$ and $\mathrm{Q}=7)$. (b) SLP as function of chain size for distinct diameter and damping factor values. (c) SLP as function of chain size for a mono-disperse system and distinct surface-to-surface distances. (d) Same as (c) for a polydisperse system. (e) Dynamic hysteresis for a mono-disperse system considering distinct surface-to-surface distances and chain size. (f) Optimum hyperthermia size ratio as function of chain size for distinct surface-to-surface distances.

characteristic times to explain the data. However, the lowest one is related to nonphysical damping values. Also, it is clear that the MNF SLP values can only be explained using the influence of the dipolar interaction for both the effective anisotropy and the damping factor parameters. In particular, the damping factor increase is related to chain formation and increased particle concentration. In this case, a surface spin-spin interaction is believed to be responsible for this enhancement. Note that the EMR data clearly corroborates such a conclusion.

On the other hand, the largest SLP values occurred for the lowest particle concentrations, which correspond also to the lowest damping values. Measurements at such low concentrations are very difficult, but we maintained those points because the heating rate of the magnetic colloid is still higher than the control (liquid carrier alone). This might indicate that the surface coating layer, which is strongly dependent upon dilution ${ }^{13}$, can influence this parameter. Note that Mn-ferrite nanoparticles are known to have strong surface-dependent magnetic properties ${ }^{46,47}$. Additional experiments are needed to better assess such an assumption. In addition, at very low field amplitude the SLP concentration dependence also low ${ }^{24}$. Nevertheless; at high field amplitudes such effect is clearly observed. At a different frequency 
(765 kHz), Boubeta et al. found significant concentration effects for spherical and cubic nanoparticles at $30 \mathrm{mT}^{24}$. In Fig. 3(d) we show also our MNF data for a lower frequency $(300 \mathrm{kHz})$, higher field amplitude value $(550 \mathrm{Oe})$, and different chain sizes (see discussion in the supplementary material - Figure S8). Our experimental results clearly indicate that SLP decreases with increasing particle concentration. The solid lines correspond to the described theoretical model but at different frequency. The chain sizes, for the high field amplitude, were estimated considering the high field (EMR) calculation. On the other hand, different from the MNF-citrate sample, the BNF starch shows no significant SLP concentration dependence. The inset in Fig. 3(d) shows the SLP as function of particle concentration for the BNF sample, at two distinct experimental situations. The explanation for such behavior can be found in Figure S9 of the supplementary material.

Thus far the calculations assumed a longitudinal configuration (field applied in the easy axis direction). In real cases such an assumption may not be realized. For randomly oriented anisotropy axes Raikher and Stepanov ${ }^{31}$ found that the new equilibrium susceptibility value decreases from the longitudinal case by a factor of 3 . If one assumes that such a term can be used in the chain model developed here one can perform similar calculations considering a variety of nanoparticle arrangements. Fig. 5 (a) shows some possible noninteracting and interacting cases in the longitudinal and random configuration neglecting chain-chain interactions. Because many distinct nanoparticle arrangements can be realized for each type of nanoparticle, and many different nanoparticle constructs can be assembled, it is interesting to explore new strategies to enhance the properties for various therapeutic applications. For instance, one might suggest that magnetoliposomes (ML) (or nanocapsules), under applied fields, are more likely to be represented by a longitudinal-type configuration, while nanospheres (NS) could be seen as the random-type of case because particle rotation could be inhibited. With that in mind calculations considering $\mathrm{Q}=3$ were performed, which was recently found experimentally for ML ${ }^{37}$. Fig. 5 (b) shows SLP as a function of the dimensionless anisotropy term for the polydisperse cases, namely: Non-interacting longitudinal (I), Non-interacting random (II), Trimer longitudinal (III) and Trimer random (IV). The same trend, as reported above, was found, i.e. a decrease in SLP due to dipolar interaction, for a lower value due to random axes configuration and a drift of optimum hyperthermia to lower particle sizes. (The latter has been recently confirmed experimentally by Lima et al. who found that isolated-type nanoparticle suspensions showed maximum SLP for a size of $18 \pm 3 \mathrm{~nm}$ that decreased and

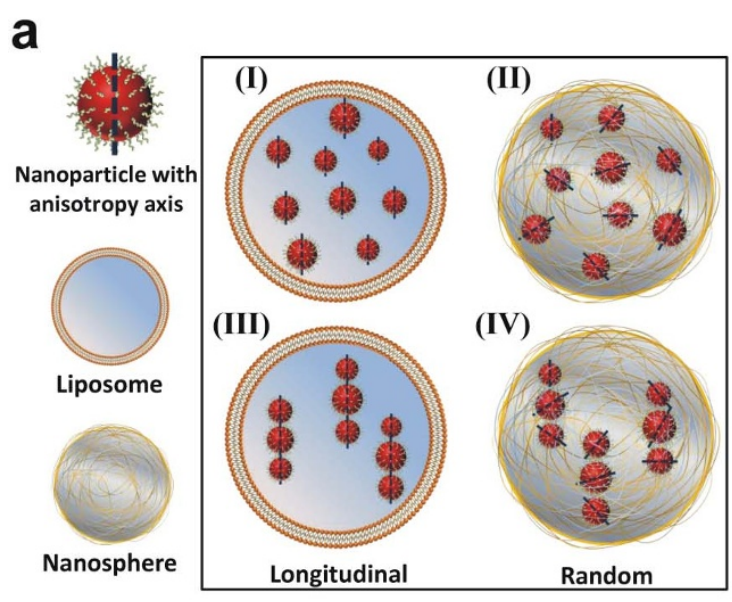

drift to a lower value $15 \pm 5 \mathrm{~nm}$ on agglomerated samples $\left.{ }^{48}\right)$. Note that we performed calculations at $100 \mathrm{kHz}$ and considered the highest possible field within the Atkinson et al. clinical field limit scaled for a treatment radius of $3.5 \mathrm{~cm}^{49,50}$. Those results can have significant consequences for clinical applications, particularly for low field amplitude biomedical applications because nanoparticles typically agglomerate inside cells $\mathrm{s}^{2,3,16,19}$ and because maximum heating might not be extracted, using low-amplitude fields, from nanoparticles having overestimated sizes. This also suggests that clinical hyperthermia procedures could be time-dependent (due to dynamic agglomerate formation in the biological media). Indeed it clearly indicates the urgent need to efficiently monitor heat deposition in-situ and also to correctly design the magnetic nanostructures for hyperthermia by taking into account the dipolar contribution to the heating phenomena. Thus active targeting and clever endocytosis strategies might be highly relevant ${ }^{51,52}$.

All of the preceding analysis was conducted using the linear response theory (LRT), which is applicable in the low-field amplitude regime. In many experimental situations, however these rules may not apply and thus additional investigation is needed. For example, it has been observed that increasing the field amplitude induces a transition to the non-linear regime. In this situation dynamic hysteresis simulations have shown that maximum SLP values occur at higher dimensionless anisotropy values with increasing field ${ }^{7,36}$. Clearly this indicates that the effect of magnitude of field amplitude is opposite to the effects of the dipole interactions (larger chain sizes). Curiously, the former shifts optimum nanoparticle sizes toward larger particle diameter ${ }^{7}$. This implies that at high-amplitude field conditions, if patient safety can be guaranteed and the particles have a non-interacting (non-agglomerate) configuration, the higher anisotropy nanoparticles having larger sizes might have a greater potential for nanoparticle hyperthermia clinical applications.

Further, our experiments were performed with magnetic colloids. In this case, depending on the nanoparticle physical parameters (for example: anisotropy, diameter, coating layer length), one might expect an additional contribution from Brownian relaxation ${ }^{11}$. Nevertheless, in real clinical situations, it has been shown that such contribution is hampered due to particle immobilization after intra-tumor injection $^{53}$. As a consequence, only the out-of-phase (intrinsic) spin rotation mechanism is expected to be relevant, which is the term (including the dipolar interaction effect) investigated in the present work. In particular, from simple effective relaxation calculations, it is easy to show that, at least for the low-barrier ("soft-like") ferrite

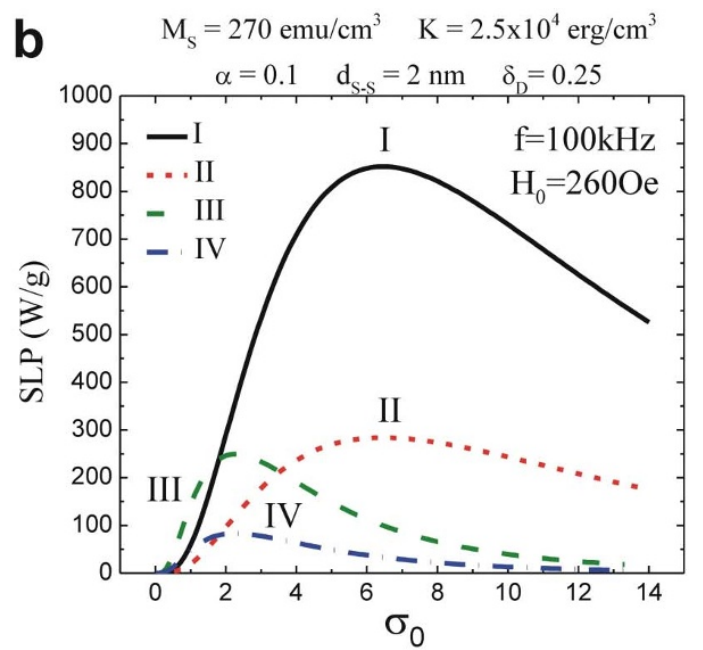

Figure $5 \mid$ Magnetic hyperthermia of distinct nanoparticle arrangements. (a) Nanoparticle arrangements inside structures I: non-interacting longitudinal case, II: non-interacting random axes, III: Chain longitudinal case and IV: Chain random axes configuration. (b) SLP as function of the dimensionless anisotropy term for distinct nanoparticle arrangements. 
nanoparticle, only the Néel-Brown relaxation mechanism is relevant for the heat generation. Indeed, from those calculations, we estimated that the Brownian relaxation would only be relevant for sizes larger than $44 \mathrm{~nm}$, if one considers the MNF sample parameters. So, regarding the heating mechanism, the colloid experiments are expected to be similar to real clinical situations. On the other hand, inside tumors, and depending on the magnetic nanocarrier delivery strategy, different particle arrangements, in comparison to linear chains, could be established. But, again, if the strong interacting and low-field amplitude conditions are maintained, similar results are expected. Also, according to our analysis, if more than one magnetic nanoparticle hyperthermia treatment procedure is necessary, one should take into account the possibility of the decrease of SLP due to distinct particle arrangements. This, on the other hand, might be relevant in planning the magnetic nanoparticle hyperthermia treatment.

In conclusion, we demonstrate that a decrease in magnetic hyperthermia efficiency is related to nanoparticle chain formation. A theoretical model taking into account the magnetic dipolar contribution showed that, in general, such term decreases the hyperthermia efficiency. Also it decreases optimum hyperthermia sizes due to self-organization. Linear response theory however has its limitations and improved models are needed to better describe physical phenomena of magnetic colloids exposed to alternating magnetic fields.

\section{Methods}

Nanoparticle synthesis. Two samples were used in this study. One was a commercial magnetite-based starch-coated nanoparticle suspension (BNF-starch S31009, micromod Partikeltechnologie, GmbH, Rostock, Germany) synthesized by highpressure homogenization according to the core-shell method ${ }^{35}$. The other colloid was synthesized by the co-precipitation procedure previously described and consisted of manganese ferrite-based (MNF-citrate) nanoparticles surface-coated with citrate ${ }^{54}$. Both colloids are dispersed in water at physiological conditions.

Nanoparticle characterization. BNF-starch transmission electron microscopy (TEM) micrographs were obtained using JEOL JEM-3010 ARP microscope operating at $300 \mathrm{kV}$ (resolution $1.7 \AA$ ), while the MNF-citrate used JEOL JEM-2100 operating at $200 \mathrm{kV}$ (resolution $2.5 \AA$ ). Standard deviation was calculated from the fitting parameters of the lognormal size distribution, i.e. $S D=\sqrt{\left(\exp \left(\delta_{D}^{2}\right)-1\right) D_{0}^{2} \exp \left(\delta_{D}^{2}\right)}$. Magnetic characterization was obtained using an ADE vibrating sample magnetometer model EV7. The electron magnetic resonance data were performed at the X-band using a Bruker ESP-300 equipment tuned at around $v=9.43 \mathrm{GHz}$.

Magnetic hyperthermia. The magnetic hyperthermia experimental set up consists basically of a power supply, a coil (inductor) and a capacitor network (refrigerant cooled $-218 \mathrm{nF}$ ), that together with the inductor (L-match) forms a resonant circuit. In our system the working frequency was around $500 \mathrm{kHz}$ and corresponds to a sinusoidal wave. The copper coil length was $10.2 \mathrm{~cm}$ with a diameter of $2.2 \mathrm{~cm}$. During the experiment the coil was cooled using a closed-loop circulating water system. At our experimental conditions the coil temperature is maintained at room temperature within an error of $0.3^{\circ} \mathrm{C}$. Measurements were performed in an interval of $300 \mathrm{~s}$. In addition, the amplitude of the alternating magnetic fields was obtained from measurements using an ac field probe bought from AMF lifesystems. The sample volume is constant and equal to $100 \mu \mathrm{L}$, while the magnetic mass is changed due to increasing the particle concentration. The samples were always inserted inside the coil at the same position. The sample container is made of polyethylene and was always the same. The container is open and we measure the temperature of the surface sample with an infrared meter. A data acquisition system converts the analogical signal from the infrared meter to a digital one that is then analyzed in a computer ${ }^{7}$. Additional high-field amplitude experiments were performed at $300 \mathrm{kHz}$ with a water-cooled 8-turn coil. The magnetic hyperthermia equipment consisted of an Ambrell system model EasyHeat-LI. In this case the temperature was measured using a fiber-optic system.

Monte carlo simulations. Simulated data are obtained using a three-dimensional poly-disperse Monte Carlo algorithm ${ }^{14}$, where the MNF-citrate sample is simulated by spherical nanoparticles. The experimental particle volume fraction range was simulated using a zeta potential of $-20.3 \mathrm{mV}$ (that corresponds to a number of molecules of $1.2 \times 10^{17} \mathrm{~mol} / \mathrm{m}^{2}$ - see Ref. 14), an external magnetic field of $0.30 \mathrm{~T}$ (close to the EMR field) and three distinct Hamaker constants, namely $\mathrm{A}=0.1,0.5$, $1.0 \times 10^{-19} \mathrm{~J}$, were considered (see supplementary information - Figure S5). Size distribution follows the experimental TEM parameters.

Model calculation. SLP numerical calculations were performed writing programs compatible with Maple 13 software. The calculations reported so far had considered the Néel-Brown contribution to uniaxial magnets, which is believed to be an excellent approximation to the MNF-citrate sample. Firstly, because of the magnetic anisotropy symmetry ${ }^{46,55}$ and second due to the fact that for this sample Brownian relaxation contribution can be ruled out. Polydisperse calculations were obtained considering a lognormal size distribution.

1. Gilchrist, R. K. et al. Selective inductive heating of lymph nodes. Annals of Surgery. 146, 596-606 (1957).

2. Jordan, A. et al. Effects of magnetic fluid hyperthermia (MFH) on C3H mammary carcinoma in vivo. Int. J. Hyperthermia 13, 587-605 (1997).

3. Thiesen, B. \& Jordan, A. Clinical applications of magnetic nanoparticles for hyperthermia. Int. J. Hyperthermia 24, 467-474 (2008).

4. Dennis, C. L. et al. Nearly complete regression of tumors via collective behavior of magnetic nanoparticles in hyperthermia. Nanotechnology 20, 395103 (2009).

5. Andrä, W., d'Ambly, C. G., Hergt, R., Hilger, I. \& Kaiser, W. A. Temperature distribution as function of time around a small spherical heat source of local magnetic hyperthermia. J. Magn. Magn. Mater. 194, 197-203 (1999).

6. Lee, J. H. et al. Exchange-coupled magnetic nanoparticles for efficient heat induction. Nature Nanotechnology 6, 418-422 (2011).

7. Verde, E. L. et al. Field dependent transition to the non-linear regime in magnetic hyperthermia experiments: Comparison between maghemite, copper, zinc, nickel and cobalt ferrite nanoparticles of similar sizes. AIP Advances 2, 032120-032142 (2012).

8. Carrey, J., Mehdaoui, B. \& Respaud, M. Simple models for dynamic hysteresis loop calculations of magnetic simple-domain nanoparticles: Application to magnetic hyperthermia optimization. J. Appl. Phys. 109, 083921-083937 (2011).

9. Kashevsky, B. E., Kashevsky, S. B. \& Prokhorov, I. V. Dynamic magnetic hysteresis in a liquid suspension of acicular maghemite particles. Particuology 7, 451-458 (2009).

10. Shliomis, M. I. \& Stepanov, V. I. Frequency dependence and long time relaxation of the susceptibility of the magnetic fluids. J. Magn. Magn. Mater. 122, 176-181 (1993).

11. Rosensweig, R. E. Heating magnetic fluid with alternating magnetic field. J. Magn. Magn. Mater. 252, 370-374 (2002).

12. Attaluri, A., Ma, R., Qiu, Y., Li, W. \& Zhu, L. Nanoparticle distribution and temperature elevations in prostatic tumours in mice during magnetic nanoparticle hyperthermia. Int. J. Hyperthermia 27, 491-502 (2011).

13. Castro, L. L. et al. Role of surfactant molecules in magnetic fluid: comparison of Monte Carlo simulation and electron magnetic resonance. Phys. Rev. E 78, 061507-061517 (2008).

14. Bakuzis, A. F., Branquinho, L. C., Castro, L. L., Eloi, M. T. A. \& Miotto, R. Chain formation and aging process in biocompatible polydisperse ferrofluids: Experimental investigation and Monte Carlo simulations. Adv. Coll. Interf. Sci. 191-192, 1-21 (2013).

15. Scholten, P. C. The origin of magnetic birefringence and dichroism in magnetic fluids. IEEE Trans. Magn. 16, 221-225 (1980).

16. Giustini, A. J., Ivkov, R. \& Hoopes, P. J. Magnetic nanoparticle biodistribution following intratumoral administration. Nanotechnology 22, 345101 (2011).

17. Hedayati, M. et al. The effect of cell cluster size on intracellular nanoparticlemediated hyperthermia: is it possible to treat microscopic tumors? Nanomedicine 8, 29-41 (2013).

18. Bordelon, D. E. et al. Magnetic nanoparticle heating efficiency reveals magnetostructural differences when characterized with wide ranging and high amplitude alternating magnetic fields. J. Appl. Phys. 109, 124904 (2011).

19. Lunov, O. et al. Modeling receptor-mediated endocytosis of polymerfunctionalized iron oxide nanoparticles by human macrophages. Biomaterials 32 , 547-555 (2011).

20. Urtizberea, A., Natividad, E., Arizaga, A., Castro, M. \& Mediano, A. Specific absorption rates and magnetic properties of ferrofluids with interaction effects at low concentrations. J. Phys. Chem. C 114, 4916-4922 (2010).

21. Tomitaka, A., Ueda, K., Yamada, T. \& Takemura, Y. Heat dissipation and magnetic properties of surface-coated $\mathrm{Fe}_{3} \mathrm{O}_{4}$ nanoparticles for biomedical applications. J. Magn. Magn. Mater. 293, 283-286 (2005).

22. Martinez-Boubeta, C. et al. Adjustable hyperthermia response of self-assembled ferromagnetic Fe-MgO core-shell nanoparticles by tuning dipole-dipole interactions. Adv. Func. Mater. 22, 3737-3744 (2012).

23. Presa, P. et al. Study of heating efficiency as a function of concentration, size and applied field in $\gamma-\mathrm{Fe}_{2} \mathrm{O}_{3}$ nanoparticles. J. Phys. Chem. C 116, 25602-25610 (2012).

24. Martinez-Boubeta, C. et al. Learning from nature to improve the heat generation of iron-oxide nanoparticles for magnetic hyperthermia applications. Sci. Rep. 3, 1652 (2013).

25. Haase, C. \& Nowak, U. Role of dipole-dipole interactions for hyperthermia heating of magnetic nanoparticle ensembles. Phys. Rev. B 85, 045435 (2012).

26. Hergt, R. et al. Magnetic properties of bacterial magnetosomes as potential diagnostic and therapeutic tools. J. Magn. Magn. Mater. 293, 80-86 (2005).

27. Hergt, R. \& Dutz, S. Magnetic particle hyperthermia-biophysical limitations of a visionary tumour therapy. J. Magn. Magn. Mater. 311, 187-192 (2007).

28. Alphandéry, E., Faure, S., Seksek, O., Guyot, F. \& Chebbi, I. Chains of magnetosomes extracted from AMB-1 magnetotactic bacteria for application in alternative magnetic field cancer therapy. ACS Nano 5, 6279-6296 (2011). 
29. Coffey, W. T. \& Kalmykov, Y. P. Thermal fluctuations of magnetic nanoparticles: Fifty years after Brown. J. Appl. Phys. 112, 121301-121347 (2012).

30. Bakuzis, A. F. \& Morais, P. C. On the origin of the surface magnetic anisotropy in manganese-ferrite nanoparticles. J. Magn. Magn. Mater. 226-230, 1924-1926 (2001).

31. Raikher, Y. L. \& Stepanov, V. I. Linear and cubic dynamic susceptibilities of superparamagnetic fine particles. Phys. Rev. B 55, 15005-15017 (1997).

32. Morup, S. \& Tronc, E. Superparamagnetic relaxation of weakly interacting particles. Phys. Rev. Lett. 72, 3278-3281 (1994).

33. Dormann, J. L. et al. Thermal variation of the relaxation time of the magnetic moment of $\gamma-\mathrm{Fe}_{2} \mathrm{O}_{3}$ nanoparticles with interparticle interactions of various strengths. Phys. Rev. B 53, 14291-14297 (1996).

34. Egli, R. Magnetic susceptibility measurements as a function of temperature and frequency I: inversion theory. Geophys. J. Int. 177, 395-420 (2009).

35. Grüttner, C. et al. Synthesis and antibody conjugation of magnetic nanoparticles with improved specific power absorption rates for alternating magnetic field cancer therapy. J. Magn. Magn. Mater. 311, 181-186 (2007).

36. Verde, E. L., Landi, G. T., Gomes, J. A., Sousa, M. H. \& Bakuzis, A. F. Magnetic hyperthermia investigation of cobalt ferrite nanoparticles: Comparison between experiment, linear response theory, and dynamic hysteresis simulations. J. Appl. Phys. 111, 123902 (2012).

37. Cintra, E. R. et al. Nanoparticle agglomerates in magnetoliposomes. Nanotechnology 20, 045103 (2009).

38. Eloi, M. T. A., Santos, J. L. Jr., Morais, P. C. \& Bakuzis, A. F. Field-induced columnar transition of biocompatible magnetic colloids: An aging study by magnetotransmissivity. Phys. Rev. E 82, 021407-021418 (2010).

39. Butter, K., Bomans, P. H. H., Frederik, P. M., Vroege, G. J. \& Philipse, A. P. Direct observation of dipolar chains in iron ferrofluids by cryogenic electron microscopy. Nature Materials 2, 88-91 (2003)

40. Smit, J. \& Beljers, H. G. Philips Res. Rep. 10, 113 (1955).

41. Jacobs, I. S. \& Bean, C. P. An approach to elongated fine-particle magnets. Phys. Rev. 100, 1060-1067 (1955).

42. Vonsovskii, S. V. in Ferromagnetic Resonance, Pergamon Press, London (1966).

43. Kurebayashi, H. et al. Uniaxial anisotropy of two-magnon scattering in an ultrathin epitaxial Fe layer on GaAs. Appl. Phys. Lett. 102, 062415 (2013).

44. Ebert, H., Mankovsky, S., Ködderitzsch, D. \& Kelly, P. J. Ab Initio calculation of the Gilbert damping parameter via the linear response formalism. Phys. Rev. Lett. 107, 066603-066606 (2011).

45. Sohn, H. \& Victora, R. H. Optimization of magnetic anisotropy and applied fields for hyperthermia applications. J. Appl. Phys. 107, 09B312 (2010).

46. Bakuzis, A. F., Morais, P. C. \& Pelegrini, F. Surface and Exchange anisotropy fields in $\mathrm{MnFe}_{2} \mathrm{O}_{4}$ nanoparticles: Size and temperature effects. J. Appl. Phys. 85, 7480-7482 (1999).

47. Vestal, C. R. \& Zhang, Z. J. Effects of surface coordination chemistry on the magnetic properties of $\mathrm{MnFe}_{2} \mathrm{O}_{4}$ spinel ferrite nanoparticles. J. Am. Chem. Soc. 125, 9828-9833 (2003)

48. Lima, E. Jr. et al. Heat generation in agglomerated ferrite nanoparticles in an alternating magnetic field. J. Phys. D: Appl. Phys. 46, 045002 (2013).

49. Atkinson, W. J., Brezovich, I. A. \& Chakraborty, D. P. Usable frequencies in hyperthermia with thermal seeds. IEEE Trans. Biomed. Eng. 31, 70-75 (1984).
50. Etheridge, M. L. \& Bischof, J. C. Optimizing magnetic nanoparticle based thermal therapies within the physical limits of heating. Annals of Biomed. Eng. 41, 78-88 (2013).

51. Decuzzi, P. \& Ferrari, M. The role of specific and non-specific interactions in receptor-mediated endocytosis of nanoparticles. Biomaterials 28, 2915-2922 (2007).

52. Petros, R. A. \& DeSimone, J. M. Strategies in the design of nanoparticles for therapeutic applications. Nature Reviews 9, 615-627 (2010).

53. Dutz, S., Kettering, M., Hilger, I., Müller, R. \& Zeisberger, M. Magnetic multicore nanoparticles for hyperthermia influence of particle immobilization in tumour tissue on magnetic properties. Nanotechnology 22, 265102 (2011).

54. Itri, R., Depeyrot, J., Tourinho, F. A. \& Sousa, M. H. Nanoparticle chain-like formation in electrical Double-layered magnetic fluids evidenced by small-angle X-ray scattering. Eur. Phys. J. E 4, 201-208 (2001).

55. Bakuzis, A. F., Morais, P. C. \& Tourinho, F. A. Investigation of the magnetic anisotropy in manganese ferrite nanoparticles using magnetic resonance. J. Magn. Reson. Ser. A 122, 100-103 (1996).

\section{Acknowledgments}

The authors would like to thank financial support from the Brazilian agencies CNPq, CAPES, FINEP, FAPEG, and FUNAPE. RI acknowledges funding provided by award number U54CA143803 from the National Cancer Institute. The content is solely the responsibility of the authors and does not necessarily represent the official views of the National Cancer Institute or the National Institutes of Health. We also thank LNNano/ CNPEM and LabMic/UFG for the use of TEM facilities.

\section{Author contributions}

N.Z. and M.S. performed the MNF-citrate synthesis. R.M. performed the Monte Carlo simulations. M.C. performed the TEM analysis of the BNF-starch, while A.C. the MNF-citrate. A.C. performed the static magnetic birefringence data and together with A.B. its analysis. L.B. performed the magnetic characterization, the electron magnetic resonance experiment and the hyperthermia experiment. A.B. and L.B. developed the theoretical model. L.B. and M.C. implemented the numerical calculations. Discussions and comments specifically related to the chain formation effect to SLP were mostly done by L.B., R.I. and A.B.. R.I. and A.B. wrote the manuscript.

\section{Additional information}

Supplementary information accompanies this paper at http://www.nature.com/ scientificreports

Competing financial interests: The authors declare no competing financial interests.

How to cite this article: Branquinho, L.C. et al. Effect of magnetic dipolar interactions on nanoparticle heating efficiency: Implications for cancer hyperthermia. Sci. Rep. 3, 2887; DOI:10.1038/srep02887 (2013).

(c) (i) $\Theta$ This work is licensed under a Creative Commons AttributionBY NG ND NonCommercial-NoDerivs 3.0 Unported license. To view a copy of this license, visit http://creativecommons.org/licenses/by-nc-nd/3.0 


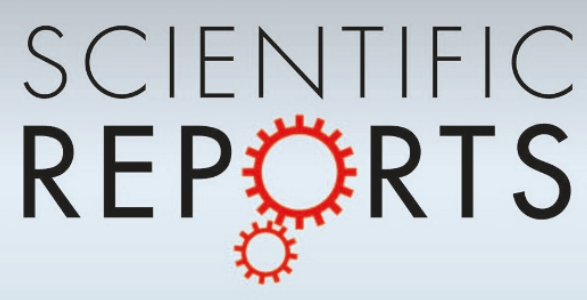

DOI: 10.1038/srep03637

SUBJECT AREAS: CORRIGENDUM: Effect of magnetic dipolar interactions on nanoparticle heating COLLOIDS NANOTECHNOLOGY IN CANCER

CANCER THERAPY MAGNETIC PROPERTIES AND MATERIALS

SCIENTIFIC REPORTS: efficiency: Implications for cancer hyperthermia

Luis C. Branquinho, Marcus S. Carrião, Anderson S. Costa, Nicholas Zufelato, Marcelo H. Sousa, Ronei Miotto, Robert Ivkov \& Andris F. Bakuzis

This Article contains a typographical error in Equation 2:

$$
\tau=\tau_{0}\left(e^{\sigma}-1\right)\left(2^{-\sigma}+\frac{2 \sigma^{3 / 2}}{\sqrt{\pi}(1+\sigma)}\right) \text { should read } \tau=\tau_{0}\left(e^{\sigma}-1\right)\left(2^{-\sigma}+\frac{2 \sigma^{3 / 2}}{\sqrt{\pi}(1+\sigma)}\right)^{-1}
$$

$3: 2887$

DOI: $10.1038 /$ srep02887

(2013)

Published:

7 October 2013

Updated:

10 January 2014 